\title{
Interesses profissionais e afetos positivos e negativos: estudo exploratório com estudantes de ensino médio
}

\author{
Ana Paula PatoNaronha - UnivesidadeSão Francisco, Itatiba, SãoPaulo Brasil \\ Camíia Santina Murgo Mansão- Faauldades Integradas deJaú, Jaú, São Paulo, Brasil
}

\begin{abstract}
Resumo
A Orientação Profissional enquanto área de intervenção e pesquisa tem buscado a compreensão de variáveis que interferem na tomada de decisão e no desenvolvimento de carreira. Trata-se de um estudo exploratório entre interesses profissionais e afetos positivos e negativos. Participaram 529 estudantes do ensino médio de escolas públicas e particulares com idades entre 14 a 27 anos $(M=16$; $D P=1,48)$. No que se refere ao sexo, $223(42,2 \%)$ eram homens e 306 (57,8\%), mulheres. Os instrumentos aplicados foram a EAZ - Escala de Afetos de Zanon e a EAP - Escala de Aconselhamento Profissional. Os participantes apresentaram maiores médias para afetos positivos, o que indica julgamento mais positivo da vida, de modo geral. A comparação entre os instrumentos revelou alguns coeficientes positivos e significativos, mas de baixa magnitude. Sugere-se que novos estudos sejam realizados com os construtos da Psicologia Positiva na orientação profissional e desenvolvimento de carreira.
\end{abstract}

Palarasdhave Psicologia positiva; Orientação profissional; Avaliação psicológica.

Professional interests and affects positive and negative: exploratory study with youngers from high school

\begin{abstract}
Professional Orientation while field of research and intervention has sought to comprehend variables which interfere in decision making and career development. This study investigates the relationship between professional interests and positive and negative affects. A total of 529 public and private high school students, 223 men (42.2\%) and 306 (57.8\%) women, ages between 14 and $27(\mathrm{M}=16$; $\mathrm{DP}=1.48)$ participated. Each volunteer was given the EAZ - Zanon Affect Scale and the EAP - Professional Counseling Scale. Participants presented higher averages in positive affects, which indicates a more positive outlook on life in general. The comparison between the scales revealed some significant positive coefficients, but of lower magnitude. It is suggested that new studies be performed with the Positive Psychology constructs in vocational orienteering and career development.
\end{abstract}

Keywards Positive psychology; Professional counseling; Psychological assessment

Intereses profesionales y afectos positivos y negativos: estudio exploratorio con estudiantes de Enseñanza Secundaria

\begin{abstract}
Resumen
La Orientación Profesional como área de intervención e investigación ha buscado entender la comprensión de variables que interfieren en las decisiones y en el desarrollo de carrera. Se trata de un estudio exploratorio entre intereses profesionales y afectos positivos y negativos. Participaron 529 estudiantes de la Enseñanza Secundaria de escuelas públicas y privadas con edades entre14 a 27 años (M=16; $\mathrm{DP}=1,48)$. En lo referente al sexo, 223 (42,2\%) eran hombres y $306(57,8 \%)$ mujeres. Los instrumentos aplicados fueron la Escala deAfetos deZanon- EAZ y la Escala deAconshlhameto Profissional - EAP. Los participantes presentaron promedios mayores para los afectos positivos, lo que indica juicio más positivo de la vida, en general. La comparación entre los instrumentos mostró algunos coeficientes positivos y significativos, pero de magnitud baja. Se sugiere que nuevos estudios sean realizados con los constructos de la Psicología Positiva en la orientación profesional y desarrollo de carrera.

Palabras dave Psicología positiva; O rientación profesional; Evaluación Psicológica.
\end{abstract}

A orientação profissional, enquanto área de conhecimento e campo de intervenção, tem se valido de algumas variáveis relevantes para a tomada de decisão, como interesses, personalidade, autoeficácia, maturidade para escolha e inteligência. No entanto, o construto mais estudado e investigado nas práticas dos orientadores tem sido os interesses profissionais (Ambiel \& Polli, 2011; Noronha \& Ambiel, 2006; Sparta, Bardagi \& Teixeira, 2006). Quanto à definição do construto, Savickas (1995), autor de referência na área, afirma que interesses são padrões de gostos e desgostos; e, posteriormente, os definiu como estado de "consciência", caracterizado por prontidão de respostas a estímulos ambientais específicos (Savickas, 1999).
Em especial, adolescentes e jovens precisam ser orientados para a transição da educação para o mundo do trabalho, de modo a ter clareza de suas habilidades e metas profissionais (Savickas, 1989). Adicionalmente, tal como pontuado por Gamboa, Paixão e Jesus (2011) as constantes mudanças do mundo do trabalho, em razão da globalização e dos avanços tecnológicos, justificam a relevância do campo.

No Brasil, nas últimas décadas, houve um aumento das pesquisas relacionadas à orientação profissional (Melo-Silva, 1999; Noronha \& Ambiel, 2006; Teixeira, Lassance, Silva \& Bargaggi, 2007, dentre outros). No que se refere aos interesses, conforme apontam os autores citados, eles têm sido muito investigados. Para Gamboa e cols. (2011), a exploração 
vocacional se constitui como um tacilitador para a escolha profissional podendo, adicionalmente favorecer a satisfação com a profissão e, em consequência, promover a manutenção da saúde mental. Uma variável ainda pouco explorada, mas que pode ser importante na compreensão das escolhas profissionais, além dos interesses, são os afetos, aqui explicados pela perspectiva da Psicologia Positiva.

Segundo Seligman (2004), a psicologia positiva é 0 estudo científico das forças e virtudes, dentre as quais encontra-se a investigação das emoções positivas. Ainda quanto à justificativa da relevância da relação entre psicologia positiva e orientação profissional e desenvolvimento de carreira, convém destacar que uma das tarefas da última é favorecer eficientes e livres inserções do indivíduo ao mundo laboral, de modo que cada sujeito tenha clareza de suas inquietações, motivações e interesses (Jafella \& Gil, 2003/ 2004).

Os afetos podem favorecer a maneira como 0 indivíduo vê a si mesmo e às outras pessoas, de modo que mais afetos positivos tendem a gerar mais prazer para vivenciar situações cotidianas. $\mathrm{O}$ afeto compõe 0 bem-estar subjetivo e tem implicações para as respostas afetivas das pessoas, incluindo afetos prazerosos e desprazerosos, a saber, afeto positivo e afeto negativo. 0 afeto positivo reflete 0 nível de entusiasmo apresentado por uma pessoa, enquanto 0 afeto negativo mostra uma dimensão geral da angústia e insatisfação, e inclui uma variedade de estados de humor aversivos, incluindo raiva, culpa, desgosto e medo (Watson, Clark \& Tellegen, 1988).

Para Ferrero e Rico (2010) as emoções positivas e negativas compreendem 0 prazer e 0 desprazer. As emoções negativas estão associadas aos eventos da vida compreendidos como ameaçadores, podendo implicar perdas e rompimento de metas. Portanto, pode-se depreender que são emoções que as pessoas não gostam de vivenciar. Já as emoções positivas associamse a eventos que são entendidos como facilitadores das metas pessoais, garantindo a sobrevivência e o bemestar. Essas emoções são agradáveis e tendem a proporcionar prazer.

Na mesma direção, Lazarus (1991) já afirmava que os afetos negativos são resultantes de uma avaliação desfavorável da pessoa em relação aos acontecimentos da vida, sendo mais frequentes os sentimentos de raiva, ansiedade, tristeza, culpa, vergonha, inveja, ciúme e desgosto. Os positivos, por sua vez, são resultado de uma avaliação positiva, sendo os sentimentos experimentados: alegria, orgulho, amor, carinho, felicidade e alívio.

De modo geral, são escassas as investigações que priorizam as medidas da psicologia positiva no âmbito da orientação profissional e do desenvolvimento de carreira. No contexto brasileiro, Scorsolini-Comin e Santos (2010) afirmaram que as pesquisas no campo ainda são incipientes e encontram-se em desenvolvimento.

Pérez (2009) investigou o papel do otimismo como preditor do nível de adaptação de jovens ingressantes do curso de psicologia de uma universidade privada. Participaram 77 estudantes, ambos os sexos, com idades entre 17 e 26 anos, com média de 19 anos. Foram utilizados o Teste de O rientação de Vida (LOT), a Escala de Otimismo de Seligman, a Escala de Satisfação $\mathrm{G}$ eral, um questionário de saúde mental positiva e um questionário sociodemográfico com informações acadêmicas. Dentre as várias análises realizadas, destaca-se a correlação entre as medidas, que gerou coeficientes significativos entre: nível de satisfação e abandono do curso $(r=-0,289)$, nível de satisfação e idade $(r=-0,490)$, nível de satisfação e gênero, em que as mulheres mostraram-se mais satisfeitas que os homens $(\mathrm{r}=0,402)$, nível de satisfação geral e a específica com a carreira $(r=0,281)$, otimismo e abandono $(r=-0,296)$.

Com vistas à construção de uma escala para a avaliação da felicidade, Alarcón (2006) elaborou 27 itens tipo Liket e os aplicou a uma amostra de 709 universitários com idades entre 20 e 30 anos. D entre os vários resultados encontrados, aqueles relacionados ao presente estudo evidenciaram índices de correlação significativos e de magnitude moderada entre felicidade e afetos positivos $(\mathrm{r}=0,48)$ e afetos negativos $(\mathrm{r}=-0,51)$.

De acordo com Alcalá, Camacho, Giner, Giner e Ibañez (2006), os estudos sobre afetos, gênero e idade têm gerado resultados contraditórios. Em razão disso, os autores estudaram 120 pessoas com idades entre 18 e 50 anos, de ambos os sexos, e aplicaram o Positiveand Negative Affeet Schetule Expande Fom (PANAS-X), em que os participantes deveriam responder à escala referindo-se "ao longo da vida" e "nas últimas semanas". Os resultados revelaram diferenças significativas entre mulheres e homens, demonstrando que elas apresentam mais felicidade e alegria, enquanto eles mostram-se mais solitários, seguros e orgulhosos. Em relação à idade, os autores organizaram dois grupos: aqueles com menos de 25 anos e os com mais de 30 anos. De modo geral, os grupos se diferenciaram e os mais jovens apresentaram mais afetos positivos e negativos que os mais velhos.

Especialmente no que se refere às diferenças entre os gêneros, Solar (2004) apresenta algumas hipóteses explicativas. Para comentar a primeira hipótese, 0 autor menciona Diener, Sandvyk e Larsen (1985), que afirmam que a intensidade afetiva corresponde a diferenças existentes entre as pessoas na intensidade que experimentam afetos de distintas qualidades. Isso 
levaria algumas pessoas a experimentarem emoções fortes, sejam positivas, sejam negativas. Essa intensidade afetiva vem sendo encontrada mais em mulheres, o que pode explicar por que apresentam em maior grau que os homens emoções negativas. A segunda hipótese das diferenças de expressão e autoperepção das emoções diz respeito à relação entre a experiência emocional que a pessoa vivencia e a forma de comunicação verbal a respeito dessa experiência. Observa-se que os homens têm um padrão mais internalizado ao mostrar suas emoções, ao passo que as mulheres parecem externalizá-las mais (Brody, 2000; Vergara \& Páez, 1989). Também parecem reconhecer melhor suas respostas emocionais que os homens. Outro dado a ser considerado é que homens e mulheres não apresentam diferenças no registro de emoções negativas experimentadas no dia a dia. Porém ao fazerem uma retrospectiva de seu estado emocional, as mulheres apresentam uma avaliação mais negativa (Barret, Robin, Pietromonaco \& Eysell, 1998).

Arteche e Bandeira (2003) realizaram um estudo que contou com uma amostra de 193 adolescentes, de ambos os sexos, 112 meninos (58\%) e 81 meninas (42\%), com idades entre 14 e 17 anos ( $M=15,7$, $\mathrm{DP}=1,0)$, que frequentavam escolas públicas. Os participantes foram divididos em três grupos, sendo um de adolescentes em regime de trabalho educativo $(\mathrm{n}=58)$, outro de adolescentes trabalhadores em regime regular $(n=58)$ e 0 terceiro grupo de adolescentes nãotrabalhadores $(\mathrm{n}=77)$. Os instrumentos utilizados foram um questionário demográfico, a Escala Multidimensional de Satisfação de Vida, as Escalas de Afeto Positivo e Afeto Negativo e a Escala de Eventos Estressores. Os resultados mostraram que os adolescentes em regime educativo mostraram-se mais satisfeitos com suas vidas em relação à subescala sdf No que diz respeito às Escalas PANAS, utilizadas para avaliar os componentes afetivos do bem-estar - afeto positivo e afeto negativo não foram encontradas correlações significativa $(r=0,12)$. Com base nas médias de cada uma das escalas, constatou-se que o escore médio do fator afeto positivo foi significativamente superior ao de afeto negativo.

O desenvolvimento da Escala Multidimensional de Satisfação de Vida para Adolescentes (ESMVA) foi o objeto de estudo de Segabinazi, Giacomini, Dias, Teixeira e Moraes (2010). 0 instrumento foi aplicado em 425 adolescentes (224 meninos e 201 meninas), com idade média de 16,1 anos ( $\mathrm{DP}=1,2)$. A análise de componentes principais possibilitou a composição final da ESMVA com 52 itens, organizados em sete componentes: família, sdf, escola, sdf comparado, nãoviolência, autoeficácia e amizade. Evidências de validade foram obtidas por meio de correlações com uma medida de autoestima. Resultados de análises univariadas para verificar possíveis diferenças entre gêneros indicaram níveis de satisfação maiores nos adolescentes do sexo masculino nos domínios família $[F(1,415)=5,13 ; \quad p=0,02]$, seff comparado $[F(1$, $415)=5,79 ; \quad p=0,02]$, não-violência $[F(1,415)=10,16$; $p<0,01]$ e autoeficácia $[F(1,415)=6,49 ; p=0,01]$. As adolescentes do sexo feminino apresentaram um maior nível de satisfação no domínio escola $[F(1,415)=2,99$; $\mathrm{p}=0,08]$.

Camargo, Abaid e Giacomini (2011) investigaram o conceito de felicidade entre adolescentes. Para tanto, foi adaptado o roteiro de entrevista semiestruturada sobre bem-estar subjetivo de crianças de Giacomoni (2002). Foram entrevistados 95 adolescentes, dos quais 48 meninas (51\%) e 47 meninos (49\%), estudantes de escolas públicas da cidade de Santa Maria-RS. A idade variou entre 12 e 20 anos $(M=14,3 ; \mathrm{DP}=1,3)$. A análise de conteúdo das respostas coletadas permitiu a elaboração de categorias que indicam que os adolescentes relacionam sentimentos abstratos e necessidades concretas ao conceito de felicidade. A análise por sexo apontou que as meninas relacionaram felicidade a variadas formas de relacionamento, enquanto os meninos, aos bens materiais e às condições de vida.

As aproximações teóricas entre 0 bem-estar subjetivo e as personalidades vocacionais de Holland foram discutidas por Gottfredson e Duffy (2008). Os autores enfatizam que as proposições da teoria vocacional versam sobre a necessidade das pessoas de buscar, inserir e persistir em ambientes profissionais congruentes com suas preferências, de modo que suas competências sejam recompensadas. Mais especialmente, as pessoas tendem a ter interesses congruentes com seus destinos educacionais ou profissionais, podendo outras influências contribuir com a tendência de ver os profissionais e os ambientes de trabalho de forma positiva, 0 que inclui as assserções relacionadas à compreensão do bem-estar subjetivo, no sentido de que as pessoas tendem a conseguir satisfação ou bem-estar.

Tal como já afirmado anteriormente, a presente pesquisa pretende investigar possíveis relações entre interesses e afetos positivos e negativos em estudantes de ensino médio. Seligman (2011) enfatizou que os conceitos da psicologia positiva, como o bem-estar, por exemplo, deveriam ser ensinados na escola, uma vez que melhoram a aprendizagem, produzem maior atenção e pensamento criativo, e previnem depressão. A asserção pode ser reforçada pelas considerações de Csikszentmihalyi (2009), de que esse novo movimento deve não apenas se preocupar com a forma como as pessoas sentem suas vidas, mas também refletir sobre a 
maneira como elas vivem e fazem suas escolhas. 0 presente trabalho consiste em um estudo exploratório com jovens em momento de escolha profissional, no qual, além das relações entre os construtos, investigar o poder de previsão dos afetos em relação às dimensões dos interesses profissionais.

\section{Participantes}

\section{Método}

Participaram da pesquisa 529 estudantes do ensino médio de cinco escolas, três públicas e duas particulares, localizadas em cidades do interior de São Paulo. D o total de participantes, 63,5\% ( $\mathrm{N}=336)$ eram estudantes do sistema particular de ensino e 35,9\% $(\mathrm{N}=190)$ do público. A faixa etária dos participantes foi de 14 a 27 anos, com uma média de 16 anos (DP= 1,48). Em relação ao sexo, 223 (42,2\%) eram homens e $306(57,8 \%)$, mulheres. No que diz respeito à distribuição dos participantes por série, 187 cursavam 0 primeiro ano (35,3\%), 164 (31\%) o segundo e 178 $(33,6 \%)$ o terceiro.

\section{Instrumentos}

A fim de atender aos objetivos propostos, foram aplicados dois instrumentos. A Escala de Aconselhamento Profissional - EAP (Noronha, Sisto \& Santos, 2007) tem como objetivo avaliar as preferências ocupacionais de jovens acima de 17 anos. E composta por 61 itens que descrevem atividades profissionais a partir de sete dimensões: Ciências Exatas, Artes e Comunicação, Ciências Biológicas e da Saúde, Ciências Agrárias e Ambientais, Atividades Burocráticas, Ciências Humanas e Sociais Aplicadas e Entretenimento. 0 examinando, ao responder, quantifica a intensidade em que ele gostaria de realizar a atividade descrita no item, numa escala tipo Liket de um a cinco pontos, variando de (5) frequentemente a desenvolveria a (1) nunca a desenvolveria.

0 estudo inicial das características psicométricas contou com a análise de 762 protocolos de estudantes, com idades que variaram ente 17 e 73 anos. Foi realizada análise a partir do modelo Rasch, que apontou índices que variaram entre 0,97-0,99 para os itens, e para as pessoas a variação se deu no intervalo de 0,76-0,88. Já na análise a partir da Teoria Clássica dos Testes, os valores de alfa ficaram entre 0,79 e 0,94, os de Speamman-Brown e Guttman entre 0,75-0,91. Em ambas as análises, os valores podem ser considerados altos, além dos resultados permitirem afirmar que as diversas dimensões do instrumento apresentaram ótima precisão.

Estudos de validade posteriores foram realizados. Como exemplo, cita-se o de Ottati (2009) e o de Sartori, Noronha e Nunes (2009). O primeiro comparou EAP com BBT-Br, por meio da aplicação em 196 estudantes dos 5ํ e 7ํㅗㄴ semestres dos cursos de Pedagogia, Odontologia e Ciência da Computação, com idades entre 19 e 49 anos $(\mathrm{M}=24,24 ; \mathrm{DP}=4,89)$, sendo $62,8 \%$ do sexo feminino. Os resultados revelaram correlações significativas entre todas as dimensões do EAP e os fatores do BBT-BR, confirmando que os instrumentos apresentam comunalidades. Em acréscimo, os cursos se diferenciaram em relação aos interesses investigados pelos instrumentos.

Por sua vez, Sartori e cols. (2009) analisaram as diferenças de médias entre a Escala de Aconselhamento Profissional (EAP) e o Seff Direted Search Caree Explorer (SD S), em relação ao sexo e série escolar. Para tanto, fizeram parte do estudo 177 jovens do Ensino Médio, com idades entre 14 e 19 anos, de quatro escolas particulares do estado de São Paulo. Os homens tiveram maiores médias na dimensão Ciências Exatas da EAP, e as mulheres nas dimensões Ciências Biológicas e da Saúde, Artes e Comunicação e Entretenimento.

A Escala de Afetos Positivos e Negativos - EAZ (Zanon \& Hutz, no prelo) é composta por 20 itens que descrevem sentimentos e emoções passadas e presentes. Trata-se de uma escala autoaplicável na qual são assinaladas respostas em uma escala tipo Liket de 5 pontos, variando de "nada a ver com você" (1 ponto) a "tudo a ver com você" (5 pontos). Sua construção se deu com base na aplicação do instrumento em 853 universitários (57\% mulheres), com média de idade de 21 anos ( $\mathrm{DP}=3)$, que responderam a 29 itens, constantes na primeira versão do instrumento.

A análise de componentes principais, com rotação Oblimin revelaram índices insatisfatórios de consistência interna para 0 modelo de três e quatro fatores, além de apresentarem diversos itens com cargas fatoriais acima de 0,35 em mais de um fator, o que fez com que a distribuição não fosse considerada pertinente. $\mathrm{Na}$ análise componencial foi encontrado o índice KaiserMeyer-Olkin de 0,90, e o teste de esfericidade de Bartlet foi significativo ( $p<0,001$ ). 0 alfa de Cronbach avaliado no estudo de consistência interna, foi de 0,83 para afeto positivo e 0,77 para afeto negativo. No estudo das correlações entre a EAZ e a Positive and Negative Affet Scheelule (PANAS) foram observados coeficientes altos de correlação entre os afetos positivos $(0,73)$ e afetos negativos $(0,74)$, indicando comunalidade entre os construtos das subescalas da EAZ e PANAS.

\section{Procedimentos}

Inicialmente 0 projeto foi encaminhado ao Comitê de Ética em Pesquisa de uma instituição de 
ensino superior para aprovação. Mediante autorização das direções das escolas, os alunos foram convidados a participar do estudo. No primeiro contato, foram esclarecidos os objetivos da pesquisa, foi solicitado que consultassem seus responsáveis quanto à participação e levassem 0 Termo de Consentimento Livre e Esclarecido para assinatura. A aplicação dos instrumentos foi coletiva, em grupos de aproximadamente 30 estudantes, com dia e horário previamente estabelecidos, em salas das escolas. 0 primeiro instrumento respondido foi a EAP e, na sequência, a EAZ, com duração média de 20 minutos. A coleta de dados teve a duração de quatro meses no período de novembro de 2010 a fevereiro de 2011.

\section{Resultados e discussão}

Serão apresentadas as estatísticas descritivas dos dois instrumentos utilizados no estudo. A Tabela 1 apresenta valores máximos e mínimos, média e DP das dimensões da EAP.

Tabela 1. Estatística descritiva da Escala de Aconselhamento Profissional ( $\mathrm{N}=529)$

\begin{tabular}{lcccc}
\hline Dimensões do EAP & Mínimo & Máximo & Média & DP \\
\hline Ciências Exatas & 1,00 & 4,86 & 2,14 & 0,83 \\
Artes e Comunicação & 1,00 & 4.86 & 2,33 & 0,83 \\
Ciências Biológicas e da Saúde & 1,00 & 5,00 & 2,38 & 1,00 \\
Ciências Agrárias e Ambientais & 1,00 & 4,69 & 2,41 & 0,83 \\
Atividades Burocráticas & 1,00 & 4.54 & 2,37 & 0,79 \\
Ciências Humanas Sociais Aplic. & 1,00 & 4,70 & 2,33 & 0,74 \\
Entretenimento & 1,00 & 5,00 & 2,24 & 0,92 \\
\hline
\end{tabular}

Convém esclarecer que para obter as médias os valores de cada dimensão foram divididos pelo total de itens que as compõem. Utilizou-se este artifício com 0 intuito de comparar as dimensões entre si, já que 0 número de itens é bastante desigual. Entre os resultados encontrados, as maiores médias foram nas dimensões Ciências Agrárias e Ambientais, Ciências Biológicas e da Saúde e Atividades Burocráticas, embora seja conveniente ressaltar a pequena diferença entre as duas últimas dimensões. Os resultados revelam que os interesses dos participantes do estudo mostraram-se diversificados, uma vez que Ciências Agrárias e Ambientais apontam para preferências associadas ao estudo do meio ambiente, ecoturismo, orientação à população sobre doenças, análise dos impactos de atividades industriais e direção de unidades de preservação ecológica. Ciências Biológicas e da Saúde têm como foco o cuidado com a saúde, enquanto as Atividades Burocráticas revelam interesses em conduzir processos de seleção, admissão e demissão, criar programas de computadores, estruturar base de dados, classificar e organizar documentos, mediar relações entre empresa e colaboradores e coordenar as operações fiscais e financeiras (Noronha $\&$ cols., 2007).
Em outra direção, as menores médias foram nas dimensões Entretenimento e Ciências Exatas, respectivamente, indicando um menor interesse dos participantes por atividades que envolvam produção e organização de desfiles e campanhas publicitárias, atividades como gerenciar hotéis, spas, clubes, flats e parques temáticos, bem como análise e interpretação de dados numéricos. Outros achados corroboram os resultados encontrados na amostra estudada nesta pesquisa. Ottati (2009) realizou um estudo com universitários, no qual foi aplicada a EAP. As maiores médias foram nas dimensões Ciências Agrárias e Ambientais, Ciências Humanas e Sociais Aplicadas e Ciências Biológicas e da Saúde. Em contrapartida, as menores médias foram nas dimensões Entretenimento e Ciências Exatas, respectivamente. Em outra investigação, com estudantes de ensino médio, Sartori, Noronha e Nunes (2009) encontraram mais preferência para as Ciências Exatas, Artes e Comunicação, enquanto 0 menor interesse foi em relação ao Entretenimento. A Tabela 2 informa sobre os valores mínimos, máximos, as médias e respectivos desvios padrão encontrados na Escala de Afetos Positivos e Negativos.

Tabela 2. Estatística descritiva da Escala de Afetos Positivos e Negativos (N=529)

\begin{tabular}{lcccc}
\hline EAPN & Mínimo & Máximo & Média & DP \\
\hline Positivos & 1,70 & 6,70 & 3,87 & 0,61 \\
Negativos & 1,00 & 4,90 & 2,69 & 0,74 \\
\hline
\end{tabular}


Os resultados revelaram que houve maior frequência de afetos positivos em detrimento dos negativos. Assim, pode-se depreender que os participantes possuem mais afetos associados a episódios intensos e frequentes de prazer. Tal como assinalado por Watson e cols. (1988), o afeto positivo é um indicador do entusiasmo apresentado por uma pessoa, enquanto o negativo revela uma dimensão geral da angústia e insatisfação. Assim, embora ambos os afetos constituam a dimensão emocional do bem-estar subjetivo, é esperado que os positivos se sobressaiam aos negativos (Seligman, 2004). D ando continuidade aos resultados, na Tabela 3 são exploradas as correlações de Pearson entre EAZ e EAP. Das 14 correlações encontradas, oito delas apresentaram coeficientes significativos, embora muito baixos.

Tabela 3. Correlações entre EAPN e as dimensões da EAP

\begin{tabular}{lllllllc}
\hline \multirow{2}{*}{ EAPN } & \multicolumn{7}{c}{ EAP } \\
\cline { 2 - 7 } & CE & AC & CBS & CAA & AB & CHS & E \\
\hline Positivos & 0,08 & 0,05 & $0,10\left(^{*}\right)$ & $0,10\left(^{*}\right)$ & $0,15\left(^{*}\right)$ & $0,10\left(^{*}\right)$ & $0,09(*)$ \\
Negativos & $0,11(*)$ & 0,08 & 0,03 & 0,01 & $0,11\left(^{*}\right)$ & 0,08 & $0,09(*)$ \\
\hline
\end{tabular}

$(*) \mathrm{p}<0,05$

$(* *) \mathrm{p}<0,001$

O s resultados da associação entre os instrumentos revelam que 0 maior coeficiente se deu entre a dimensão Atividades Burocráticas e os afetos positivos. Os afetos negativos correlacionaram-se mais fortemente com Ciências Exatas e Atividades Burocráticas. Os achados indicam pouca comunalidade entre os construtos, embora algum registro de relação entre eles, tal como esperado teoricamente. Mais especialmente, interesses podem ser compreendidos como padrões de gostos e desgostos (Savickas, 1995) e afetos compõem o bem-estar subjetivo, e são compostos por afetos prazerosos (afeto positivo) e desprazerosos (afeto negativo) (Watson \& cols., 1988).

As Atividades Burocráticas podem ser ilustradas por gerenciar serviços; participar de processos de seleção, admissão e demissão; estruturar e manter base de dados; classificar e organizar documentos; e conduzir relações entre empresa e empregados. Entretenimento tem como atividades: atender hóspedes, associados e turistas em hotéis, spase clubes; promover a instalação de hotéis; e gerenciar flats pousadas, hotéis, parques temáticos. facilitadores das metas pessoais, garantindo a sobrevivência e o bemestar. Assim, pode-se compreender que as atividades descritas gerararam tanto emoções agradáveis na amostra estudada, quanto desagradáveis (Ferreira \& Rico, 2010).

Em outra medida, os afetos negativos também estão associados com as atividades: analisar e interpretar dados numéricos; planejar e implantar linhas automatizadas de produção alimentícia; criar programas de computadores; e projetar satélites e foguetes, características das Ciências Exatas. Assim, angústia e insatisfação, e uma variedade de estados de humor aversivos estão mais associados com este tipo de preferência.

No que diz respeito à previsão da dimensão de Ciências Exatas, (Tabela 4) 0 coeficiente de determinação $\left(\mathrm{R}^{2}\right)$ ajustado foi de $0,02 \quad(\mathrm{~F}(2,497)=$ 5,348; p<0,005). A análise do coeficiente de determinação ajustado indicou $2 \%$ da variância. Para a dimensão Artes e Comunicação e Ciências Biológicas e da Saúde, assim como nas demais, utilizou-se um modelo do qual constaram os afetos positivos e negativos. $O \mathrm{R}^{2}$ ajustado foi de 0,009 para ambas, sendo $(F(2,497)=3,157 ; p<0,043)$ para a primeira e $(F(2,498)=3,275 ; p<0,039)$ para a segunda. No que diz respeito à previsão da dimensão Ciências Agrárias e Ambientais, 0 coeficiente de determinação $\left(R^{2}\right)$ ajustado foi de 0,008 $(F(2,497)=2,955 ; p<0,053)$, portanto, não significativo.

Atividades Burocráticas foi a dimensão que apresentou maior capacidade de predição, com um $\left(\mathrm{R}^{2}\right)$ ajustado de 0,04, embora ainda baixo $(F(2,490)=$ 10,671; p<0,000). Os achados, de alguma forma, reforçam os dados da análise de correlação, na qual a mesma dimensão se associou com igual magnitude tanto com afetos positivos, quanto com negativos. Ciências Humanas e Sociais Aplicadas $(\mathrm{F}(2,500)=$ 5,956; $p<0,003)$ e Entretenimento $(F(2,502)=5,093$; $\mathrm{p}<0,006)$ apresentaram o mesmo $\left(\mathrm{R}^{2}\right)$ ajustado que Ciências Exatas $(0,02)$. 
Tabela 4. Coeficientes da regressão linear para prever as dimensões de interesses profissionais

\begin{tabular}{|c|c|c|c|c|c|c|}
\hline \multirow{2}{*}{\multicolumn{2}{|c|}{ Dimensões }} & \multicolumn{2}{|c|}{ Coeficientes não-padronizados } & \multirow{2}{*}{$\begin{array}{l}\text { Coeficientes padronizados } \\
\text { Bea }\end{array}$} & \multirow{2}{*}{$\mathrm{t}$} & \multirow{2}{*}{$\mathrm{p}$} \\
\hline & & $\mathrm{B}$ & DP & & & \\
\hline Ciências & Constante & 1,193 & 0,308 & & 3,876 & 0,000 \\
\hline \multirow[t]{2}{*}{ Exatas } & Positivos & 0,014 & 0,006 & 0,107 & 2,323 & 0,021 \\
\hline & Negativos & 0,014 & 0,005 & 0,129 & 2,803 & 0,005 \\
\hline Artes & Constante & 1,621 & 0,306 & & 5,296 & 0,000 \\
\hline \multirow[t]{2}{*}{ Comunicação } & Positivos & 0,010 & 0,006 & 0,077 & 1,682 & 0,093 \\
\hline & Negativos & 0,011 & 0,005 & 0,102 & 2,219 & 0,027 \\
\hline Ciếncias & Constante & 1,465 & 0,367 & & 3,989 & 0,000 \\
\hline \multirow[t]{2}{*}{ Biológicas } & Positivos & 0,018 & 0,007 & 0,113 & 2,451 & 0,015 \\
\hline & Negativos & 0,008 & 0,006 & 0,059 & 1,293 & 0,196 \\
\hline Ciências & Constante & 1,723 & 0,308 & & 5,591 & 0,000 \\
\hline \multirow[t]{2}{*}{ Agrárias } & Positivos & 0,015 & 0,006 & 0,112 & 2,425 & 0,016 \\
\hline & Negativos & 0,004 & 0,005 & 0,036 & 0,776 & 0,438 \\
\hline Atividades & Constante & 1,045 & 0,290 & & 3,606 & 0,000 \\
\hline \multirow[t]{2}{*}{ Burocráticas } & Positivos & 0,023 & 0,006 & 0,184 & 4,024 & 0,000 \\
\hline & Negativos & 0,016 & 0,005 & 0,147 & 3,204 & 0,001 \\
\hline Ciências & Constante & 1,408 & 0,270 & & 5,221 & 0,000 \\
\hline \multirow[t]{2}{*}{ Humanas } & Positivos & 0,016 & 0,005 & 0,134 & 2,934 & 0,003 \\
\hline & Negativos & 0,011 & 0,005 & 0,113 & 2,472 & 0,014 \\
\hline \multirow[t]{3}{*}{ Entretenimento } & Constante & 1,173 & 0,337 & & 3,476 & 0,001 \\
\hline & Positivos & 0,018 & 0,007 & 0,121 & 2,635 & 0,009 \\
\hline & Negativos & 0,014 & 0,006 & 0,110 & 2,407 & 0,016 \\
\hline
\end{tabular}

\section{Considerações finais}

A presente pesquisa teve um caráter exploratório e pretendeu investigar as relações entre interesses profissionais e afetos positivos e negativos em um grupo de jovens em idade de transição escola-trabalho. Além disso, análises de regressão foram realizadas.

A justificativa para um estudo dessa natureza centra-se sobre duas vertentes. O momento de transição, tal como pontuado por vários autores (Gamboa \& cols., 2011; Savickas, 1989; Sparta \& cols., 2006; dentro outros), caracteriza-se por um período de acentuado conflito, no qual o jovem se vê com a responsabilidade de uma decisão. De acordo com Jafella e Gil (2003/2004), a orientação profissional pode favorecer que 0 ingresso do jovem ao mundo laboral se dê de maneira eficiente, de modo que ele tenha conhecimento de suas inquietações, motivações e interesses. Ademais, para Seligman (2004), a Psicologia Positiva é 0 estudo científico das forças e virtudes, dentre as quais se encontra a investigação das emoções positivas e, se ensinada nas escolas, facilitaria não apenas a aprendizagem, mas evitaria a instalação de depressão e outros males. Ainda de acordo com 0 autor, durante a adolescência é comum ser vivenciada uma crise em razão das mudanças físicas e emocionais, 0 que pode provocar no adolescente uma visão das coisas a partir do imediato, sem clara perspectiva de futuro.

Quanto aos achados do estudo, foram encontradas modestas, mas algumas associações entre interesses e afetos, de modo que tal dado não deve ser descartado, e sim aprofundado em pesquisas futuras. Os dados aqui obtidos são iniciais e restringem-se a uma amostra de jovens de uma localização do estado de São Paulo. Novas pesquisas com instrumentos e participantes diferenciados podem favorecer a melhor compreensão das relações aqui investigadas.

\section{Referências}

Alarcón, R. (2006). Desarrollo de uma escala factorial para medir la felicidad. Revista Interamericana de Psicdoja, 40(1), 99-106.

Alcalá, V., Camacho, M., Giner, D., Giner, J., \& Ibañez, E. (2006). Afectos y gênero. Psicthema, 18(1), 143-148.

Ambiel, R. M., \& Polli, M. F. (2011) Análise da produção cientifica brasileira sobre avaliação psicológica em orientação profissional. Esurbs Intedisciplinares emPsicoga, Londina, 2(1), 103-121.

Arteche, A. X., \& Bandeira, D. R. (2003). Bem-estar subjetivo: um estudo com adolescentes 
trabalhadores. PsicoUSF, 8(2), 193-201.

Barret, L. F., Robin, L., Pietromonaco, P. R., \& Eyssel, K. M. (1998). Are woman the "more emotional sex"? Evidence from emotional experiences in social context. CognitionandEmtion, 12, 555-578.

Brody, L. (2000). The socialization of gender differences in emotional expression: displey rules, infant temperament and differentiation. Em A. Fisher (Ed.), Gender and emtion: soial psychdojical perspetives (pp. 24-47). Nova Iorque: Cambridge University Press.

Camargo, S. P. H., Abaid, J. L. W., \& Giacomini, C. H. (2011). D o que eles precisam para serem felizes? A felicidade na visão de adolescentes. Revista Semestral da Associação Brasileira de Psicoloja Escolar e Eduracional, 15(2), 241-250.

Csikszentmihalyi, M. (2009). The promise of positive psychology. Psychdogical Topiss, 18(2), 203-211.

Diener, E., Sandivyk E., \& Larsen, R. J. (1985). Age and sex effects for emotional intensity. Dexdqpmental Psydology, 21, 542-546.

Ferrero, I. T., \& Rico, T. P. (2010). Psicologia positiva y promocion de la salud mental, emoções positivas y negativas. Em A. C. Vañó (Org.), Aplicaciones Edurativas de La Psicdoja Positiva (pp. 130-140). Hispania: G eneralitat Valenciana.

Gamboa, V., Paixão, M. P., \& Jesus, S. N. (2011). A eficácia de uma intervenção de carreira para a exploração vocacional. Revista Brasileira de OriettaçãoProfissional, 12(2), 153-164.

Giacomoni, C. (2002). Bemetar subjeivo infantil: concito de felicidade e construção de instrumentos para avaliaçãa (Tese de D outorado). Universidade Federal do Rio Grande do Sul, Rio Grande do Sul, Brasil.

Gottfredson, G. D ., \& Duffy, R. D. (2008). Using a theory of vocational personalities and work environments to explore subjective well-being. Jaumal of Career Assessmet, 16(1), 44-59.

Jafella, S. A., \& Gil, D. V. (2003/ 2004). Orientación vocacional y desarrollo de competencias. Orietacióny Sociedad, 4, 1-8.

Lazarus, R. S. (1991). Emtion and adaptation Nova Iorque: Oxford University Press.

Melo-Silva, L. L. (1999). A formação do orientador profissional. Reista daABOP, 3(1), 161-165.

Noronha, A. P. P., \& Ambiel, R. A. M. (2006). Orientação profissional e vocacional: análise da produção científica. PsicoUSF, 11(1), 75-84.
Noronha, A. P., Sisto, F., \& Santos, A. A. A. (2007). Escala deAconsdhamento Profissional - EAP. Manual técnico. São Paulo: Vetor.

Ottati, F. (2009). Escala de Aconsdhamento Profissional (EAP) e BBT-BR: estudb de eidênia de validade (Dissertação de Mestrado). Universidade São Francisco, São Paulo, Brasil.

Pérez, C. L. (2009). O ptimismo y salud positive como predictors de la adaptación a la vida universitaria. Ada Codonhiama dePsicdoǵa, 12(1), 95-107.

Sartori, F. A, Noronha, A. P. P., \& Nunes, M. F. O. (2009). Comparações entre EAP e SD S: interesses profissionais em alunos de ensino médio. Bdeimde Psicloga, 59(130), 17-29.

Savickas, M. L. (1989). Career style assessment and counseling. Em T. Sweeney (Org.), Adleian cansding a practical approach for a new decade ( $\mathrm{pp}$. 289-320). Muncie: Accelerated D evelopment.

Savickas, M. L. (1995). Examining the personal meaning of inventoried interests during career counseling. Jarmal of Career Assessment, 3(2), 188201.

Savickas, M. L. (1999). Examining the personal meaning of inventoried interests during career couseling. Jaumal of Career Assesment, 3, 188201.

Sartori, F. A., Noronha, A. P. P., \& Nunes, M. F. N. (2009). Comparações entre EAP e SD S: interesses profissionais em alunos do ensino médio. Bdetim dePsicloga, 130, 17-29.

Scorsolini-Comin, F., \& Santos, M. A. (2010). Psicologia positiva e os instrumentos de avaliação no contexto brasileiro. Psicdogia: Rellexão e Cńtica, 23(3), 440-448.

Seligman, M. E. P. (2004). Feiicidade autêtica: usandb a nova psicloga positiva para a relização pemante Rio de Janeiro, RJ: O bjetiva.

Seligman, M. E. P. (2011). Flarish Nova Iorque: Free Press.

Segabinazi, J. D., Giacomini, C. H., Dias, A. C. G., Teixeira, M. A. P., \& Moraes, D. A. O. (2010). Desenvolvimento e validação preliminar de uma escala multidimensional de satisfação de vida para adolescentes. Psicoloja: Teria ePesquisa, 26(4), 653659.

Solar, F. C. (2004). Diferencias de gênero em bienestar y malestar emocional: evidencias contradictorias. Teapia Psiclógica, 22(2), 165-169. 
Sparta, M., Bardagi, M. P., \& Teixeira, M. A. P. (2006). Modelos e instrumentos de avaliação em orientação profissional: perspectiva histórica e situação no Brasil. Reiista Brasilera de Oriettação Profissional, 7(2), 19-32.

Teixeira, M. A. P., Lassance, M. C. P., Silva, B. M. B., \& Bargaggi, M. P. (2007). Produção científica em orientação profissional: uma análise da Revista Brasileira de Orientação Profissional. Reista Brasilera deOrietacăão Profissional, 8(2), 25-40.

Vergara, A., \& Páez, D . (1989). Rol sexual e diferencias em vivencia emocional: explicaciones psicológicosociales. Em A. Echervarria \& D. Páez (Eds.), Emmions Pespeetivas Psicossodales (pp. 235-257). Madri: Fundamentos.
Watson, D., Clark, L. A., \& Tellegen, A. (1988). Developmental and validation of brief measures of positive and negative affect: the PANAS scales. Jarmal of Pesconality and Social Psydhology, 54, 10631070.

Zanon, C., \& Hutz, C. S. (no prelo). Escala de Afteos Zanon

Sobre as autoras:

Ana Paula Porto Nononha é psicóloga, doutora em Psicologia Ciência e Profissão pela Pontifícia Universidade Católica de Campinas, docente do Programa de Pós-Graduação Striło Sensu em Psicologia pela Universidade São Francisco e bolsista produtividade em pesquisa pelo CNPq.

Camélia Santina Murgo Mansão é psicóloga, doutora em Psicologia Ciência e Profissão pela Pontifícia Universidade Católica de Campinas, docente das Faculdades Integradas de Jaú e da Universidade do O este Paulista. Bolsista de pós-doc pelo CNPq.

\section{Contato com as autoras:}

Rua Alexandre Rodrigues Barbosa, 45 - Centro - CEP: 13.251-900. Itatiba-SP.

E-mail: ana.noronha@ usf.edu.br 